AERO-ASTRONAUTICS REPORT NO. 22

\title{
BODIES OF MAXIMUM LIFT-TO-DRAG RATIO IN HYPERSONIC FLOW
}

\author{
by
}

ARTHUR H. LUSTY, Jr. and ANGELO MIELE
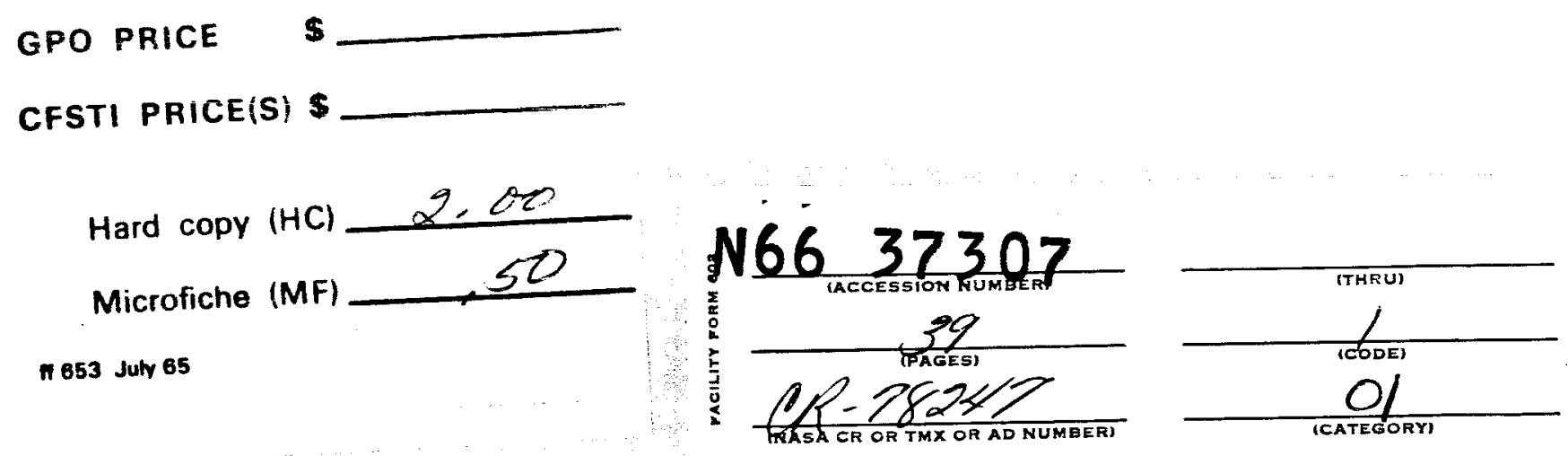

RICE UNIVERSITY

1966 
BODIES OF MAXIMUM LIFT-TO-DRAG RATIO

IN HYPERSONIC FLOW $^{(*)}$

by

ARTHUR H. LUSTY, Jr. ${ }^{(*)}$ and ANGELO MIELE ${ }^{(* *)}$

$\underline{\text { SUMMARY }}$

The problem of maximizing the lift-to-drag ratio of a slender, flat-top body of semicircular cross section in hypersonic flow is investigated with the indirect methods of the calculus of variations. The pressure distribution is assumed to be modified Newtonian and a constant value of the skin-friction coefficient is employed.

First, unconstrained configurations are considered, and it is found that the optimum body is a half-cone whose vertex angle is unique but whose size is undetermined. The scaling factor determining the size of the body can be found providing one geometric constraint is imposed on either the length, the thickness, or the volume.

Next, configurations are considered upon which two geometric constraints are imposed, that is, given thickness and length, given volume and length, and given

${ }^{(*)}$ This research was supported by the Langley Research Center of the National Aeronautics and Space Administration under Grant No. NGR-44-006-034.

${ }^{(* *)}$ Research Associate in Astronautics, Department of Mechanical and Aerospace Engineering and Materials Science, Rice University, Houston, Texas.

$\left(^{* * *}\right)$ Professor of Astronautics and Director of the Aero-Astronautics Group, Department of Mechanical and Aerospace Engineering and Materials Science, Rice University, Houston, Texas . 
volume and thickness. For each case, the lift-to-drag ratio parameter, the thickness ratio parameter, and the optimum shape are presented as functions of a single similarity parameter involving the two given quantities, the skin-friction coefficient, and the constant modifying the Newtonian pressure distribution. 


\section{INTRODUCTION}

Currently, it is of interest to develop long range vehicles capable of cruising or gliding at hypersonic speeds and maneuverable vehicles capable of reentering the atmosphere (Refs. 1 and 2). In order to increase the range and the maneuverability, the vehicle should be designed with high aerodynamic efficiency, that is, high lift-to-drag ratio. Thus, we are presented with an optimization problem: that of maximizing the lift-to-drag ratio for various constraints imposed on the geometric dimensions.

In this area of problems, previous studies were carried out in Refs. 3 and 4 for the class of slender, flat-top, homothetic bodies. Direct methods were used, and the analysis was confined to the case where the longitudinal contour is represented by a power law. In this report, the above restriction is removed, and the indirect methods of the calculus of variations are employed in order to optimize bodies of arbitrary longitudinal contour. While the cross section is assumed to be semicircular, this restriction is by no means essential; the results of this report can be used to generate those valid for a body of arbitrary cross section by employing the similarity law of Ref. 5 .

The complete list of hypotheses is as follows: (a) a plane of symmetry exists between the left-hand and right-hand sides of the body; (b) the upper surface is flat; (c) the body is slender in the longitudinal sense; (d) the transversal contour is semicircular; (e) the free-stream velocity is parallel to the line of intersection of the plane of symmetry and the plane of the flat top; (f) the pressure coefficient is proportional to the cosine squared of the angle formed by the free-stream velocity and the normal to each surface element; $(\mathrm{g})$ the base drag is neglected; (h) the skin-friction coefficient 
is constant and equal to a suitably chosen average value; and (i) the contribution of the tangential forces to the lift is negligible with respect to the contribution of the normal forces. 


\section{FUNDAMENTAL EQUATIONS}

In order to relate the drag and the lift of a body to its geometry, we define the following cylindrical coordinate system $\operatorname{Oxr} \theta$ (Fig. 1): the origin $O$ is the apex of the body; $\mathrm{x}$ is a coordinate measured along the line of intersection of the plane of symmetry and the flat top, positive toward the base; $r$ is the distance of any point from the axis of symmetry; and $\theta$ measures the angular position of the vector $\vec{r}$ with respect to the plane of the flat top.

If the hypotheses (a) through (i) are invoked and if the lower surface is represented by the relationship $r=r(x)$, the drag $D$ and the lift $L$ per unit free-stream dynamic pressure q can be written as (Ref. 3)

$$
\begin{aligned}
& D / q=\int_{0}^{l} r\left[2 \pi n \dot{r}^{3}+(2+\pi) C_{f}\right] d x \\
& L / q=\int_{0}^{l} 4 n r \dot{r}^{2} d x
\end{aligned}
$$

where $\ell$ denotes the length of the body, $\mathrm{n}$ a factor modifying the Newtonian pressure distribution ${ }^{(*)}, C_{f}$ the skin-friction coefficient, and $\dot{r}$ the derivative $d r / d x$. As a consequence, the lift-to-drag ratio is given by

$$
\frac{\pi L}{2 D}=\frac{\int_{0}^{l} r \dot{r}^{2} d x}{\int_{0}^{l} r\left(\dot{r}^{3}+K\right) d x}
$$

where

$$
K=(2+\pi) C_{f} / 2 \pi n
$$

$\left(^{*}\right)$ The pressure coefficient employed in Eqs. (1) is $C_{p}=2 n \dot{r}^{2}$. 
This lift-to-drag ratio is to be maximized subject to the isoperimetric constraint of given volume

$$
2 \mathrm{~V} / \pi=\int_{0}^{l} \mathrm{r}^{2} \mathrm{dx}
$$

and the inequality constraint

$$
\dot{\mathbf{r}} \geq 0
$$

which expresses the limit of validity of the Newtonian pressure law.

One way to account for Ineq. (5) is to transform it into an equality constraint by introducing an appropriate auxiliary variable. If this is done, subarcs $\dot{\mathbf{r}}=0$ are likely to appear in the optimum contour. From previous experience (see Chapter 14 of Ref. 6), it is known that these subarcs start at the initial point and terminate at the final point. Therefore, an alternate way to account for Ineq. (5) is to investigate the class of bodies preceded by a spike and followed by a cylinder and, therefore, defined by (Fig. 2)

$$
\begin{array}{llll}
\dot{r}=0 & , & & 0 \leq x \leq x_{i} \\
\dot{r} \geq 0 & r_{i} \leq r \leq r_{f} & , & x_{i} \leq x \leq x_{f} \\
\dot{r}=0 & , &
\end{array}
$$

where the initial abscissa $x_{i}$, the final abscissa $x_{f}$, the length $\ell$, and the thickness $t$ may be either prescribed or free. If this point of view is taken, the variational problem reduces to that of maximizing the expression

$$
\frac{\pi L}{2 D}=\frac{\int_{x_{i}}^{x_{f}} r \dot{r}^{2} d x}{\int_{x_{i}}^{x_{f}} r\left(\dot{r}^{3}+K\right) d x+K t\left(l-x_{f}\right)}
$$


subject to the isoperimetric constraint

$$
2 V / \pi=\int_{x_{j}}^{x_{f}} r^{2} d x+t^{2}\left(l-x_{f}\right)
$$

and certain prescribed boundary conditions. 


\section{NECESSARY CONDITIONS}

According to Ref. 7, the proposed problem is equivalent to that of maximizing the functional

$$
I=\int_{x_{i}}^{x_{f}} F(r, \dot{x}, E, \lambda) d x+G\left(x_{f}, l, t, E, \lambda\right)
$$

with respect to the functions $\mathrm{r}(\mathrm{x})$ and the parameters $\ell$ and $t$ satisfying the isoperimetric constraint (8) and the prescribed boundary conditions. In Eq. (9), the functions $F$ and $G$ are defined as

$$
\begin{aligned}
& F=r \dot{r}^{2}-E r\left(\dot{r}^{3}+K\right)+\lambda r^{2} \\
& G=-E K t\left(l-x_{f}\right)+\lambda t^{2}\left(l-x_{f}\right)
\end{aligned}
$$

where

$$
E=\pi L / 2 D
$$

is a multiplier proportional to the unknown maximum lift-to-drag ratio and $\lambda$ is a multiplier associated with the volume constraint. Both $E$ and $\lambda$ are constant during the optimization process.

Euler Equation. According to standard variational procedures (see, for instance, Chapter 1 of Ref. 6), the optimum shape must satisfy the Euler equation

$$
\mathrm{dF}_{\dot{\mathrm{r}}} / \mathrm{dx}-\mathrm{F}_{\mathrm{r}}=0
$$

which admits the first integral

$$
\mathrm{F}-\dot{\mathrm{r}} \mathrm{F}_{\dot{\mathrm{r}}}=\mathrm{C}
$$

since the fundamental function $(10-1)$ does not contain the independent variable explicitly. 
The explicit form of Eq. (13) is given by

$$
\left(2 E \dot{r}^{3}-\dot{r}^{2}-E K\right) r+\lambda r^{2}=C
$$

Transversality Condition. The integration constants that appear in the solution of the Euler equation must be determined from the transversality condition

$$
\left[C \delta \mathbf{x}+F_{\dot{r}} \delta \mathbf{r}\right]_{\mathbf{i}}^{\mathbf{f}}+\delta G=0
$$

which must be satisfied identically for every set of variations consistent with the prescribed end conditions. Since $\delta r_{i}=0$ and $\delta r_{f}=\delta t$, the explicit form of Eq. (15) is given by

$$
\begin{aligned}
-C \delta x_{i} & +\left(C-\lambda t^{2}+E K t\right) \delta x_{f}+\left(\lambda t^{2}-E K t\right) \delta \ell \\
& +\left[t \dot{r}_{f}\left(2-3 E \dot{r}_{f}\right)+(2 \lambda t-E K)\left(\ell-x_{f}\right)\right] \delta t=0
\end{aligned}
$$

which for the four classes of solutions,

Class I:

Class II:

Class III:

Class IV:

$$
x_{i}=0, r_{i}=0
$$$$
\mathrm{x}_{\mathrm{f}}=\ell, \mathrm{r}_{\mathrm{f}}=\mathrm{t}
$$$$
x_{i}>0, r_{i}=0
$$$$
\mathrm{x}_{\mathrm{i}}=0, \mathrm{r}_{\mathrm{i}}=0
$$$$
x_{i}>0, r_{i}=0
$$$$
\mathrm{x}_{\mathrm{f}}=\ell, \mathrm{r}_{\mathrm{f}}=\mathrm{t}
$$$$
\mathrm{x}_{\mathrm{f}}<\ell, \mathrm{r}_{\mathrm{f}}=\mathrm{t}
$$$$
x_{f}<l, r_{f}=t
$$

yields the following natural boundary conditions: 
$x_{i} \equiv$ free:

$$
\mathrm{C}=0
$$

$\underline{x_{f} \equiv \text { free: }}$

$$
C-\lambda t^{2}+E K t=0
$$

$\ell \equiv$ free:

$$
\lambda t^{2}-E K t=0
$$

$\underline{\mathrm{x}_{\mathrm{f}}=\ell \equiv \text { free: }}$

$$
\mathrm{C}=0
$$

$\underline{t} \equiv$ free:

$$
t \dot{r}_{f}\left(2-3 E \dot{r}_{f}\right)+(2 \lambda t-E K)\left(\ell-x_{f}\right)=0
$$

Legendre Condition. In order to insure that the solutions of the Euler equation are optimal with respect to weak variations in the slope, it is necessary that the Legendre condition

$$
\mathrm{F}_{\dot{r} \dot{r}} \leq 0
$$

be satisfied. Its explicit form is given by

$$
\dot{\mathrm{r}} \geq 1 / 3 \mathrm{E}
$$

Weierstrass Condition. If strong variations in the slope are considered, the Legendre condition is to be replaced by the Weierstrass condition

$$
F\left(r, \dot{r}_{c}, E, \lambda\right)-F(r, \dot{r}, E, \lambda)-F_{\dot{r}}(r, \dot{r}, E, \lambda)\left(\dot{r}_{c}-\dot{r}\right) \leq 0
$$

where $r$ and $\dot{r}$ are the ordinate and the slope of the extremal arc and $\dot{r}_{c}$ is the slope of the comparison arc. The explicit form of this inequality

$$
\mathrm{r}\left(\dot{\mathrm{r}}_{\mathrm{c}}-\dot{\mathrm{r}}\right)^{2}\left(1-2 E \dot{\mathrm{r}}-\mathrm{E}_{\mathrm{c}}\right) \leq 0
$$

is satisfied for every choice of the comparison slope consistent with the constraint (5) providing

$$
\dot{\mathrm{r}} \geq 1 / 2 \mathrm{E}
$$

As expected, the Weierstrass condition is more restrictive than the Legendre condition. 


\section{NONDIMENSIONAL EQUATIONS}

Before analyzing particular cases, it is convenient to introduce the nondimensional

coordinates

$$
\xi=x / l \quad, \quad \rho=r / t
$$

and the nondimensional quantities

$$
\begin{aligned}
& \mathrm{E}_{*}=\mathrm{EK}^{1 / 3}=(\pi / 2)(\mathrm{L} / \mathrm{D}) \mathrm{K}^{1 / 3} \\
& \mathrm{~T}_{*}=\tau \mathrm{K}^{-1 / 3}=(\mathrm{t} / \ell) \mathrm{K}^{-1 / 3} \\
& \lambda_{*}=\lambda \mathrm{tK} \mathrm{K}^{-2 / 3} \\
& \mathrm{C}_{*}=(\mathrm{C} / \mathrm{t}) \mathrm{K}^{-2 / 3} \\
& \mathrm{~V}_{*}=\left(2 \mathrm{~V} / \pi \ell^{3}\right) \mathrm{K}^{-2 / 3} \\
& \mathrm{~V}_{\mathrm{o}}=\left(2 \mathrm{~V} / \pi \mathrm{t}^{3}\right) \mathrm{K}^{1 / 3}
\end{aligned}
$$

where $\tau$ is the thickness ratio. With the aid of these definitions, Eqs . (7) and (8) can be rewritten as

$$
\begin{aligned}
& \int_{\xi_{i}}^{\xi_{f}} \rho P(\dot{\rho}) d \xi+E_{*}\left(1-\xi_{f}\right)=0 \\
& V_{*} / T_{*}^{2}=V_{o} T_{*}=\int_{\xi_{i}}^{\xi_{f}} \rho^{2} d \xi+1-\xi_{f}
\end{aligned}
$$

where

$$
P(\dot{p})=E_{*} \tau_{*}^{3.3} \dot{p}^{3}-\tau_{*}^{2} \dot{p}^{2}+E_{*}
$$


and where $\dot{\rho}$ denotes the derivative $d \rho / d \xi$. The first integral (14) becomes

$$
\lambda_{*} \rho^{2}+Q(\dot{\rho}) \rho-C_{*}=0
$$

where

$$
Q(\dot{\rho})=2 E_{*} \tau_{*}^{3} \dot{p}^{3}-\tau_{*}^{2} \dot{\rho}^{2}-E_{*}
$$

and

$$
\dot{\rho} \geq 1 / 2 \mathrm{E}_{*} \tau_{*}
$$

owing to the Weierstrass condition (23). Finally, the boundary conditions (17) and (18) become

Class I:

$$
\xi_{i}=0, \quad \rho_{i}=0 \quad, \quad \xi_{f}=1, \rho_{f}=1
$$

Class II:

$$
\xi_{i}>0, \quad \rho_{i}=0 \quad, \quad \xi_{f}=1, \rho_{f}=1
$$

Class III:

$$
\varepsilon_{i}=0, \rho_{i}=0 \quad, \quad \varepsilon_{f}<1, \rho_{f}=1
$$

Class IV:

$$
\xi_{i}>0, \rho_{i}=0 \quad, \quad \xi_{f}<1, \quad \rho_{f}=1
$$

and

$$
\begin{aligned}
& x_{i} \equiv \text { free: } \\
& \mathrm{C}_{*}=0 \\
& \underline{x_{f} \equiv \text { free: }} \\
& \mathrm{C}_{*}-\lambda_{*}+\mathrm{E}_{*}=0 \\
& \ell \equiv \text { free: } \\
& \lambda_{*}-E_{*}=0 \\
& \mathrm{x}_{\mathrm{f}}=\ell \equiv \text { free: } \\
& \mathrm{C}_{*}=0 \\
& \underline{t} \equiv \text { free: } \\
& \tau_{*}^{2} \dot{\rho}_{f}\left(2-3 E_{*} \tau_{*} \dot{\rho}_{f}\right)+\left(2 \lambda_{*}-E_{*}\right)\left(1-\varepsilon_{f}\right)=0
\end{aligned}
$$




\section{PARTICULAR CASES}

With the aid of expressions (26) through (32), the following particular cases are analyzed: (a) unconstrained configuration; (b) given thickness and length; (c) given volume and length; and (d) given volume and thickness. For each particular case, we first determine the solutions of Class I; then, if more solutions are needed, we investigate those of Class II, Class III, and Class IV. For problems where the length is free, the following concept is relevant: one may add a spike of zero thickness to each extremal solution of Class I and generate an infinite number of solutions of Class II having the same lift-to-drag ratio; analogously, one may add a spike of zero thickness to each extremal solution of Class III and generate an infinite number of solutions of Class IV having the same lift-to-drag ratio. Therefore, if the length is free, only solutions of Class I and Class III shall be analyzed. 


\section{UNCONSTRAINED CONFIGURATION}

If the length, the thickness, and the volume are free, the analysis shows that the extremal solution is of Class I and is governed by the first integral (28) which must be solved in conjunction with the condition $\lambda_{*}=0$ and the boundary conditions $(31-1)$, $(32-4)$, and (32-5). Therefore, Eq. (28) reduces to

$$
Q(\dot{0})=0
$$

which implies that

$$
\dot{\rho}=\mathrm{C}_{1}
$$

and, upon integration, that

$$
\rho=\mathrm{C}_{1} \varepsilon+\mathrm{C}_{2}
$$

Because of Eqs. (31-1), the constants in Eq. (35) take the values

$$
\mathrm{C}_{1}=1 \quad, \quad \mathrm{C}_{2}=0
$$

so that

$$
\rho=\varepsilon
$$

meaning that the optimum flat-top body is a semicone. The lift-to-drag ratio parameter and the thickness ratio parameter are given by

$$
E_{*}=\sqrt[3]{4} / 3 \quad, \quad T_{*}=\sqrt[3]{2}
$$

as can be seen by employing Eqs. (31-1), (32-5), (33), and (37). 
Notice that the geometry of the optimum body is completely determined in the $\varepsilon_{0}$-coordinate system but depends on a scaling factor in the xr-coordinate system. Therefore, there exist an infinite number of bodies having the lift-to-drag ratio parameter (38-1). However, if one geometric quantity is specified (the length, the thickness, or the volume), the optimum body becomes unique. Should two or three geometric quantities be simultaneously specified, the geometry of the optimum body would generally change, and a loss in the lift-to-drag ratio parameter would occur with respect to that predicted by Eq. $(38-1)$. 


\section{GIVEN THICKNESS AND LENGTH}

If the thickness and the length are given while the volume is free, the extremal solution is governed by the first integral (28) which must be solved in conjunction with the condition $\lambda_{*}=0$ and the boundary conditions (31) and (32). This first integral can be rewritten as

$$
\rho=C_{*} / Q(\dot{0})
$$

and its solutions are of Class I or Class II depending on the value of the thickness ratio parameter $\tau_{*}$, a known quantity.

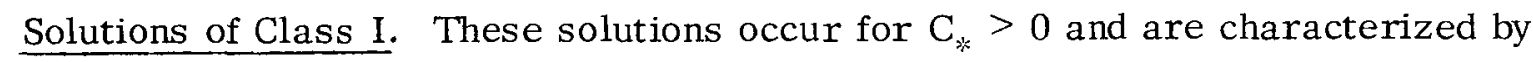
the fact that the optimum body behaves as a $3 / 4$-power body near the axis of symmetry so that $\dot{p}_{i}=\infty$. By applying Eq. (39) at the final point, we see that

$$
C_{*}=Q\left(\dot{0}_{f}\right)
$$

and, as a consequence,

$$
\mathrm{o}=\mathrm{Q}\left(\dot{\mathrm{o}}_{\mathrm{f}}\right) / \mathrm{Q}(\dot{\mathrm{o}})
$$

Next, we observe that $\mathrm{d} \xi=\mathrm{d} \rho / \dot{\rho}$ and, upon integration, we obtain the relationship

$$
\varepsilon=Q\left(\dot{o}_{f}\right) \int_{\dot{\rho}}^{\infty}\left[Q^{\prime}(\dot{o}) / \dot{o}^{2}(\dot{o})\right] d \dot{\rho}
$$

(Q' denotes the derivative $d Q / d \dot{o})$, which--together with $\mathrm{Eq} \cdot(41)$--describes the optimum shape in parametric form. The lift-to-drag ratio parameter $E_{*}$ and the final slope $\dot{0}_{f}$ are unknown and must be determined in terms of the thickness ratio parameter $\tau_{*}$. 
To do so, we apply Eq. (42) at the final point to obtain the relationship

$$
1-Q\left(\dot{\rho}_{f}\right) \int_{\dot{\rho}_{f}}^{\infty}\left[Q^{\prime}(\dot{\rho}) / \dot{p} Q^{2}(\dot{\rho})\right] d \dot{\rho}=0
$$

Also, we combine Eqs. (26-1), (41), and (42) and deduce that

$$
\int_{\dot{p}}^{\infty}\left[P(\dot{\rho}) Q^{\prime}(\dot{o}) / \dot{\rho} Q^{3}(\dot{\rho})\right] d \dot{\rho}=0
$$

Since the functions $P$ and $Q$ contain the quantities $E_{*}$ and $\tau_{*}$, the relations (43) and (44) define the functions

$$
E_{*}=f_{1}\left(\tau_{*}\right) \quad, \quad \dot{o}_{f}=f_{2}\left(\tau_{*}\right)
$$

implicitly. As a consequence, the parametric equations of the optimum shape (41) and (42) become

$$
\rho=f_{3}\left(\dot{\rho}, \tau_{*}\right) \quad, \quad \xi=f_{4}\left(\dot{p}, \tau_{*}\right)
$$

and, upon elimination of $\dot{\rho}$, allow us to describe the extremal solution in the form

$$
\rho=f_{5}\left(\xi, T_{*}\right)
$$

Equations (45-1) and (47) have been evaluated numerically and are valid providing (Figs. 3 and 4)

$$
\tau_{*}>\sqrt[3]{2}
$$

Solutions of Class II. These solutions occur for $\mathrm{C}_{*}=0$ and are characterized by the fact that the initial slope is finite. Since the first integral (39) is solved by

$$
Q(\dot{p})=0
$$


Eqs. (34) and (35) hold providing

$$
C_{1}=1 /\left(1-\xi_{i}\right) \quad, \quad C_{2}=-\xi_{i} /\left(1-\xi_{i}\right)
$$

Clearly, these solutions include a spike of zero thickness followed by a semicone described by

$$
\rho=\left(\xi-\xi_{\mathbf{i}}\right) /\left(1-\xi_{\mathbf{i}}\right)
$$

In order to determine the lift-to-drag ratio parameter $\mathrm{E}_{*}$ and the initial abscissa $\xi_{i}$, Eq. (51) must be combined with Eqs . (26-1) and (49) to yield the relationships

$$
\mathrm{E}_{*}=\sqrt[3]{4} / 3 \quad, \quad \xi_{\mathrm{i}}=1-\tau_{*} / \sqrt[3]{2}
$$

Therefore, upon elimination of $\xi_{\mathbf{i}}$ from Eqs. (51) and (52-2), the optimum shape can be rewritten as

$$
0=1-\left(\sqrt[3]{2} / T_{*}\right)(1-\xi)
$$

Equations (52-1) and (53) are plotted in Figs. 3 and 4 and are valid providing

$$
T_{*} \leq \sqrt[3]{2}
$$




\section{GIVEN VOLUME AND LENGTH}

If the volume and the length are given while the thickness is free, the extremal solution is governed by the first integral (28) which must be solved in conjunction with the isoperimetric constraint (26-2) and the boundary conditions (31) and (32). The analysis shows that the solutions of Eq. (28) are of Class I or Class II depending on the value of the volume-length parameter $\mathrm{V}_{*}$, a known quantity.

Solutions of Class I. These solutions occur for $\mathrm{C}_{*}>0, \lambda_{*}>0$ and are characterized by the fact that the optimum body behaves as a 3/4-power body near the axis of symmetry so that $\dot{p}_{i}=\infty$. If the first integral (28) is regarded to be an algebraic equation of the second degree in $\rho$, the only relevant solution is given by

$$
\rho=\left(1 / 2 \lambda_{*}\right) R(\dot{\rho})
$$

where

$$
R(\dot{p})=-Q(\dot{p})+\sqrt{Q^{2}(\dot{p})+\epsilon}
$$

and $\epsilon=4 \mathrm{C}_{*} \lambda_{*}$. By applying Eq. (55) at the final point, we see that

$$
2 \lambda_{*}=R\left(\dot{p}_{f}\right)
$$

and, as a consequence,

$$
p=R(\dot{\rho}) / R\left(\dot{\rho}_{f}\right)
$$

Next, we observe that $d \bar{S}=d \rho / \dot{p}$ and, upon integration, we obtain the relationship

$$
\xi=\left[1 / R\left(\dot{\rho}_{f}\right)\right] \int_{\infty}^{\dot{\rho}}(1 / \dot{\rho}) R^{\prime}(\dot{\rho}) d \dot{\rho}
$$


( $R^{\prime}$ denotes the derivative $\left.d R / d \rho\right)$, which--together with $E q \cdot(58)$--describes the optimum shape in parametric form. The lift-to-drag ratio parameter $E_{*}$, the thickness ratio parameter $\tau_{*}$, the final slope $\dot{\rho}_{f}$, and the constant $€$ are unknown and must be determined in terms of the volume-length parameter $V_{*}$. To do so, we apply Eq. (59) at the final point to obtain the relationship

$$
R\left(\dot{\rho}_{f}\right)-\int_{\infty}^{\dot{\rho}_{f}}(1 / \dot{\rho}) R^{\prime}(\dot{\rho}) d \dot{\rho}=0
$$

Also, we combine Eqs. (26), (58), and (59) and deduce that

$$
\begin{array}{r}
\int_{\infty}^{\dot{\rho}_{f}}(1 / \dot{\rho}) P(\dot{\rho}) R(\dot{\rho}) R^{\prime}(\dot{\rho}) d \dot{\rho}=0 \\
V_{*} R^{3}\left(\dot{\rho}_{f}\right)-\tau_{*}^{2} \int_{\infty}^{\dot{\rho}_{f}}(1 / \dot{\rho}) R^{2}(\dot{\rho}) R^{\prime}(\dot{\rho}) d \dot{\rho}=0
\end{array}
$$

Finally, we rewrite the boundary condition (32-5) in the following form:

$$
3 \mathrm{E}_{* ;} \tau_{*} \dot{\mathrm{p}}_{\mathrm{f}}-2=0
$$

Since the functions $\mathrm{P}$ and $\mathrm{R}$ contain the quantities $\mathrm{E}_{*}, \tau_{*}$, and $\epsilon$, the relations (60) through (62) define the functions

$$
E_{*}=f_{1}\left(V_{*}\right), \quad \tau_{*}=f_{2}\left(V_{*}\right), \quad \dot{\rho}_{f}=f_{3}\left(V_{*}\right), \quad \epsilon=f_{4}\left(V_{*}\right)
$$

implicitly. As a consequence, the parametric equations of the optimum shape (58) and (59) become

$$
\rho=\mathrm{f}_{5}\left(\dot{\rho}, \mathrm{V}_{*}\right) \quad, \quad \xi=\mathrm{f}_{6}\left(\dot{\rho}, \mathrm{V}_{*}\right)
$$


and, upon elimination of $\dot{p}$, allow us to describe the extremal solution in the form

$$
\rho=f_{7}\left(\varepsilon, V_{* ;}\right)
$$

Equations (63-1), (63-2), and (65) have been evaluated numerically and are valid providing (Figs. 5 through 7)

$$
\mathrm{V}_{*}>\sqrt[3]{4} / 3
$$

Solutions of Class II. These solutions occur for $\mathrm{C}_{*}=\lambda_{*}=0$ and are characterized by the fact that the initial slope is finite. Once more, the first integral (28) is solved by

$$
Q(\dot{\rho})=0
$$

so that Eqs. (34), (35), (50), and (51) hold. In order to determine the lift-to-drag ratio parameter $E_{*}$, the thickness ratio parameter $\tau_{*}$, and the initial abscissa $\xi_{i}$, Eq. (51) must be combined with Eqs. (26) and (67) to yield the relationships

$$
\begin{aligned}
& \mathrm{E}_{*}=\sqrt[3]{4} / 3 \\
& \tau_{*}=\left(3 \sqrt[3]{2} \mathrm{~V}_{*}\right)^{1 / 3} \\
& \xi_{i}=1-\left(3 \mathrm{~V}_{*} / \sqrt[3]{4}\right)^{1 / 3}
\end{aligned}
$$

Therefore, upon elimination of $\varepsilon_{i}$ from Eqs. (51) and (68-3), the optimum shape can be rewritten as

$$
\rho=1-\left(\sqrt[3]{4} / 3 V_{*}\right)^{1 / 3}(1-\xi)
$$

Equations (68-1), (68-2), and (69) are plotted in Figs. 5 through 7 and are valid providing

$$
\mathrm{V}_{*} \leq \sqrt[3]{4} / 3
$$




\section{GIVEN VOLUME AND THICKNESS}

If the volume and the thickness are given while the length is free, the extremal solution is governed by the first integral (28) which must be solved in conjunction with the is operimetric constraint (26-2) and the boundary conditions (31) and (32). The analysis shows that the solutions of Eq. (28) are of Class I or Class III depending on the value of the volume-thickness parameter $\mathrm{V}_{\mathrm{o}}$, a known quantity.

Solutions of Class I. For these solutions, which are characterized by $\mathrm{C}_{*}=0$ and $\lambda_{*} \leq \mathrm{E}_{*}$, the first integral (28) reduces to

$$
0=\left(-1 / \lambda_{*}\right) Q(\dot{0})
$$

By applying this equation at the end points, we see that the initial and final slopes satisfy the relationships

$$
\mathrm{Q}\left(\dot{0}_{\dot{i}}\right)=0 \quad, \quad Q\left(\dot{\rho}_{f}\right)+\lambda_{*}=0
$$

so that

$$
\rho=Q(\dot{\rho}) / Q\left(\dot{\rho}_{f}\right)
$$

Next, we observe that $\mathrm{d} \xi=\mathrm{d} \rho / \dot{\rho}$ and, upon integration, we obtain the relation

$$
\varepsilon=\left[1 / \mathrm{Q}\left(\dot{\rho}_{\mathrm{f}}\right)\right] \int_{\dot{\rho}_{\dot{i}}}^{\dot{\rho}}(1 / \dot{\rho}) Q^{\prime}(\dot{\rho}) d \dot{\rho}
$$

which--together with Eq. (73)--describes the optimum shape in parametric form. The lift-to-drag ratio parameter $E_{*}$, the thickness ratio parameter $\tau_{*}$, the initial slope $\dot{\rho}_{i}$, and the final slope $\dot{\rho}_{f}$ are unknown and must be determined in terms of the volume-thickness parameter $\mathrm{V}_{\mathrm{o}}$. To do so, we apply Eq. (74) at the final point to obtain the relationship 


$$
Q\left(\dot{\rho}_{f}\right)-\int_{\dot{\rho}_{i}}^{\dot{\rho}_{f}}(1 / \dot{\rho}) Q^{\prime}(\dot{\rho}) d \dot{\rho}=0
$$

Also, we combine Eqs. (26), (73), and (74) and deduce that

$$
\begin{array}{r}
\int_{\dot{\rho}_{i}}^{\dot{\rho}_{f}}(1 / \dot{\rho}) P(\dot{\rho}) Q(\dot{\rho}) Q^{\prime}(\dot{\rho}) d \dot{\rho}=0 \\
V_{o}{ }^{T} * Q^{3}\left(\dot{\rho}_{f}\right)-\int_{\dot{\rho}_{i}}^{\dot{\rho}_{f}}(1 / \dot{\rho}) Q^{2}(\dot{\rho}) Q^{\prime}(\dot{\rho}) d \dot{\rho}=0
\end{array}
$$

Since the functions $P$ and $Q$ contain $E_{*}$ and $\tau_{*}$, the relations (72-1), (75), and (76) define the functions

$$
E_{*}=f_{1}\left(V_{0}\right), \tau_{*}=f_{2}\left(V_{o}\right), \dot{\rho}_{i}=f_{3}\left(V_{o}\right), \dot{\rho}_{f}=f_{4}\left(V_{o}\right)
$$

implicitly. As a consequence, the parametric equations of the optimum shape (73) and (74) become

$$
\rho=\mathrm{f}_{5}\left(\dot{\rho}, \mathrm{V}_{0}\right) \quad, \quad \xi=\mathrm{f}_{6}\left(\dot{\rho}, \mathrm{V}_{0}\right)
$$

and, upon elimination of $\dot{p}$, allow us to describe the extremal solution in the form

$$
\mathrm{p}=\mathrm{f}_{7}\left(\xi, \mathrm{V}_{\mathrm{o}}\right)
$$

Equations (77-1), (77-2), and (79) have been evaluated analytically and are valid providing (Figs. 8, 9, and 11)

$$
V_{0} \leq 0.313
$$


As Fig. 11 shows, the optimum shape is slightly concave for $\mathrm{V}_{0}<0.265$, is conical for $\mathrm{V}_{\mathrm{O}}=0.265$, and is slightly convex for $0.265<\mathrm{V}_{\mathrm{o}} \leq 0.313$.

Solutions of Class III. For these solutions, which are characterized by $\mathrm{C}_{*}=0$ and $\lambda_{*}=\mathrm{E}_{*}$, the shape equations (73) and (74) are still valid. The lift-to-drag ratio parameter $E_{*}$, the thickness ratio parameter $\tau_{*}$, the initial slope $\dot{p}_{i}$, the final slope $\dot{\rho}_{f}$, and the transition abscissa $\xi_{f}$ can be determined in terms of the volume-thickness parameter $\mathrm{V}_{\mathrm{o}}$ by solving the relationships

$$
\begin{array}{r}
Q\left(\dot{o}_{i}\right)=0 \\
Q\left(\dot{\rho}_{f}\right)+E_{*}=0 \\
\varepsilon_{f} Q\left(\dot{\rho}_{f}\right)-\int_{\dot{\rho}_{i}}^{\dot{\rho}_{f}}(1 / \dot{\rho}) Q^{\prime}(\dot{\rho}) d \dot{\rho}=0 \\
E_{*}\left(1-\varepsilon_{f}\right) Q^{2}\left(\dot{o}_{f}\right)+\int_{\dot{\rho}_{i}}^{\dot{\rho}_{f}}(1 / \dot{0}) P(\dot{\rho}) Q(\dot{\rho}) Q^{\prime}(\dot{\rho}) d \dot{\rho}=0 \\
\left(V_{o} \tau_{*}-1+\varepsilon_{f}\right) Q^{3}\left(\dot{\rho}_{f}\right)-\int_{\dot{\rho}_{i}}^{\rho_{f}}(1 / \dot{\rho}) Q^{2}(\dot{o}) Q^{\prime}(\dot{\rho}) d \dot{\rho}=0
\end{array}
$$

which admit solutions of the form (77) and

$$
\varepsilon_{\mathrm{f}}=\mathrm{f}_{8}\left(\mathrm{~V}_{\mathrm{o}}\right)
$$

Once more, the optimum shape is described by equations of the form (78) which, upon elimination of $\dot{j}$, yield the functional relation (79). Equations (77-1), (77-2), (82), and (79) have been evaluated analytically and are valid providing (Figs . 8 through 11)

$$
V_{\mathrm{o}} \geq 0.313
$$




\section{DISCUSSION AND CONCLUSIONS}

In the previous sections, the problem of maximizing the hypersonic lift-to-drag ratio of a slender, flat-top body of semicircular cross section is investigated with the indirect methods of the calculus of variations. The pressure distribution is assumed to be modified Newtonian and a constant value of the skin-friction coefficient is employed. The main conclusions of the analysis are as follows:

(a) The optimum unconstrained configuration is a semicone having a lift-to-drag ratio parameter $\mathrm{E}_{*}=0.529$, a thickness ratio parameter $\tau_{*}=1.26$, a volume-length parameter $\mathrm{V}_{*}=0.529$, and a volume-thickness parameter $\mathrm{V}_{\mathrm{o}}=0.265$. For $\mathrm{n}=1$ and $\mathrm{C}_{\mathrm{f}}=10^{-3}$, these quantities correspond to $\mathrm{L} / \mathrm{D}=3.6, \mathrm{t} / \ell=0.118, \mathrm{~V} / \ell^{3}=0.00727$, and $\mathrm{V} / \mathrm{t}^{3}=4.44$. However, the size of the semicone is undetermined.

(b) If either the length, the thickness, or the volume is prescribed, the aerodynamic and geometric characteristics are identical with those of case (a), but the size of the optimum body is uniquely determined.

(c) If the thickness and the length are given, two solutions are possible depending on the value of the thickness ratio parameter. For $\tau_{*} \leq 1.26$, the optimum body consists of a spike followed by the semicone of case (a). For $\tau_{*}>1.26$, the optimum body is convex, it behaves as a 3/4-power body in the neighborhood of the axis of symmetry and, therefore, has an infinite slope at the nose.

(d) If the volume and the length are given, two solutions are possible depending on the value of the volume-length parameter. For $V_{: \leftarrow} \leq 0.529$, the optimum body consists of a spike followed by the semicone of case (a). For $V_{*}>0.529$, the optimum body is convex, it behaves as a 3/4-power body in the neighborhood of the axis of symmetry 
and, therefore, has an infinite slope at the nose.

(e) If the volume and the thickness are given, three solutions are possible depending on the value of the volume-thickness parameter. For $V_{0} \leq 0.265$, the optimum body is slightly concave. For $0.265 \leq \mathrm{V}_{\mathrm{o}} \leq 0.313$, the optimum body is slightly convex. Finally, for $\mathrm{V}_{\mathrm{o}} \geq 0.313$, the optimum body is composed of a convex portion followed by a cylindrical portion. Regardless of the value of $V_{0}$, all of the solutions have a sharp nose.

In closing, the following comments are in order:

(f) If the similarity law of Ref. 5 is invoked, the solutions of this report can be employed in order to generate those valid for a cross-sectional shape other than semicircular. If this is done, the lift-to-drag ratio of the unconstrained configuration can be improved by . $2 \%$ using the best elliptical cross section, $12.5 \%$ using the best sinusoidal cross section, and $47 \%$ using the best triangular cross section (Ref. 3 ).

(g) Since the present optimum bodies are convex or only slightly concave, we feel that the use of the Newtonian pressure law is justified.

(h) While this analysis neglects the dependence of the skin-friction coefficient on the body shape during the optimization process, this point of view is justified in the light of the results of Ref. 8 . 


\section{REFERENCES}

1. BECKER, J.V., Studies of High Lift/Drag Ratio Hypersonic Configurations, Paper presented at the 4th ICAS Congress, Paris, France, 1964.

2. FETTERMAN, D.E., HENDERSON, A., Jr., BERTRAM, M.H., and JOHNSTON, P.J., Studies Relating to the Attainment of High Lift-Drag Ratios at Hypersonic Speeds, NASA TN No. D-2956, 1965.

3. MIELE, A., Lift-to-Drag Ratios of Slender Bodies at Hypersonic Speeds, Journal of the Astronautical Sciences, Vol. 13, No. 1, 1966.

4. MIELE, A. and HUANG, H.Y., Power-Law Bodies of Maximum Lift-to-Drag Ratio in Hypersonic Flow, Rice University, Aero-Astronautics Report No. 20, 1966.

5. MIELE, A., Similarity Laws for Bodies Maximizing the Lift-to-Drag Ratio at Hypersonic Speeds, Journal of the Astronautical Sciences, Vol. 13, No. 2, 1966.

6. MIELE, A., Editor, Theory of Optimum Aerodynamic Shapes, Academic Press, New York, 1965.

7. MIELE, A., The Extremization of Products of Powers of Functionals and Its Application to Aerodynamics, Astronautica Acta, Vol. 12, No. 1, 1966.

8. MIELE, A. and LUSTY, A.H., Jr., On Optimum Wedges and Semicones in Hypersonic Viscous Flow, Rice University, Aero-Astronautics Report No. 28, 1967. 
LIST OF CAPTIONS

Fig. 1 Coordinate system.

Fig. 2 Generalized meridian contour.

Fig. 3 Maximum lift-to-drag ratio.

Fig. 4 Optimum shape.

Fig. 5 Maximum lift-to-drag ratio.

Fig. 6 Optimum thickness ratio.

Fig. 7 Optimum shape.

Fig. 8 Maximum lift-to-drag ratio.

Fig. 9 Optimum thickness ratio.

Fig. 10 Transition abscissa.

Fig. 11 Optimum shape. 


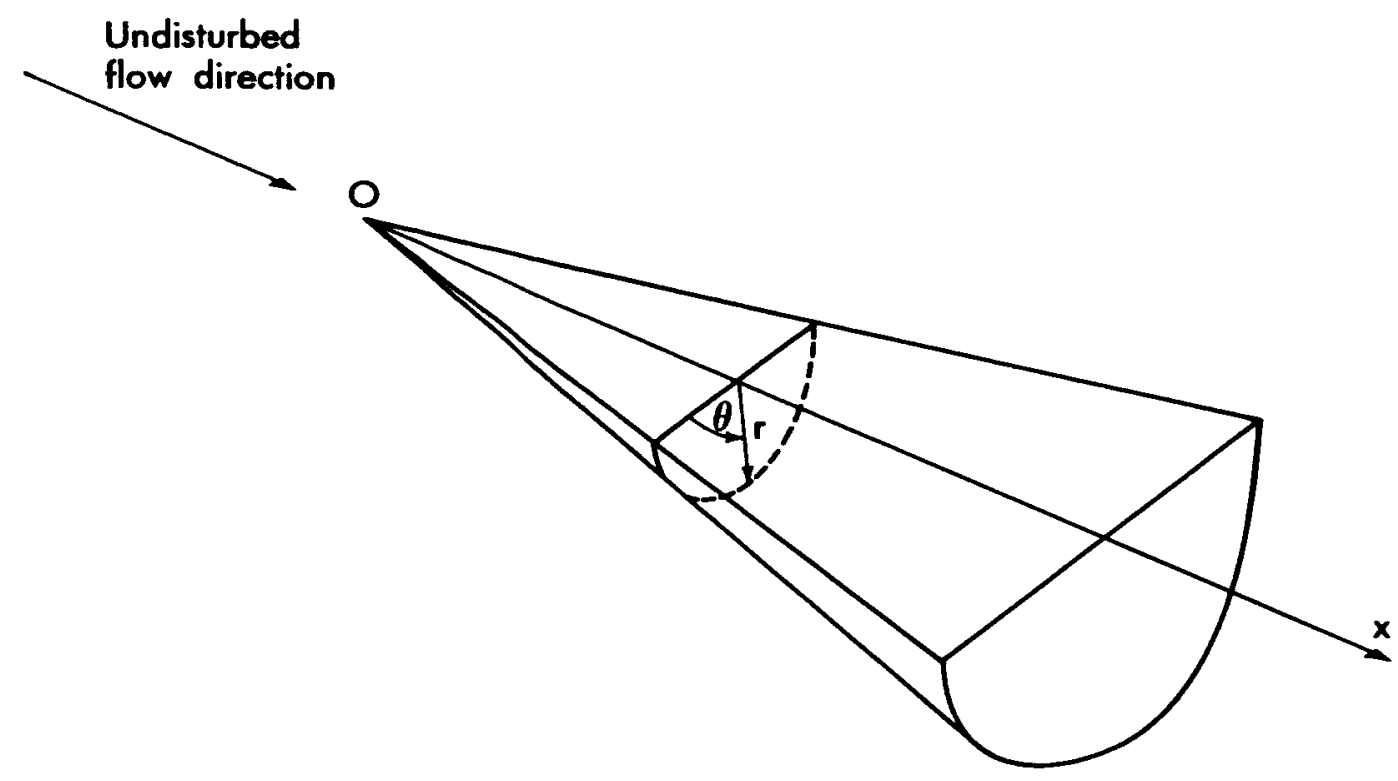

Fig. 1 Coordinate system.

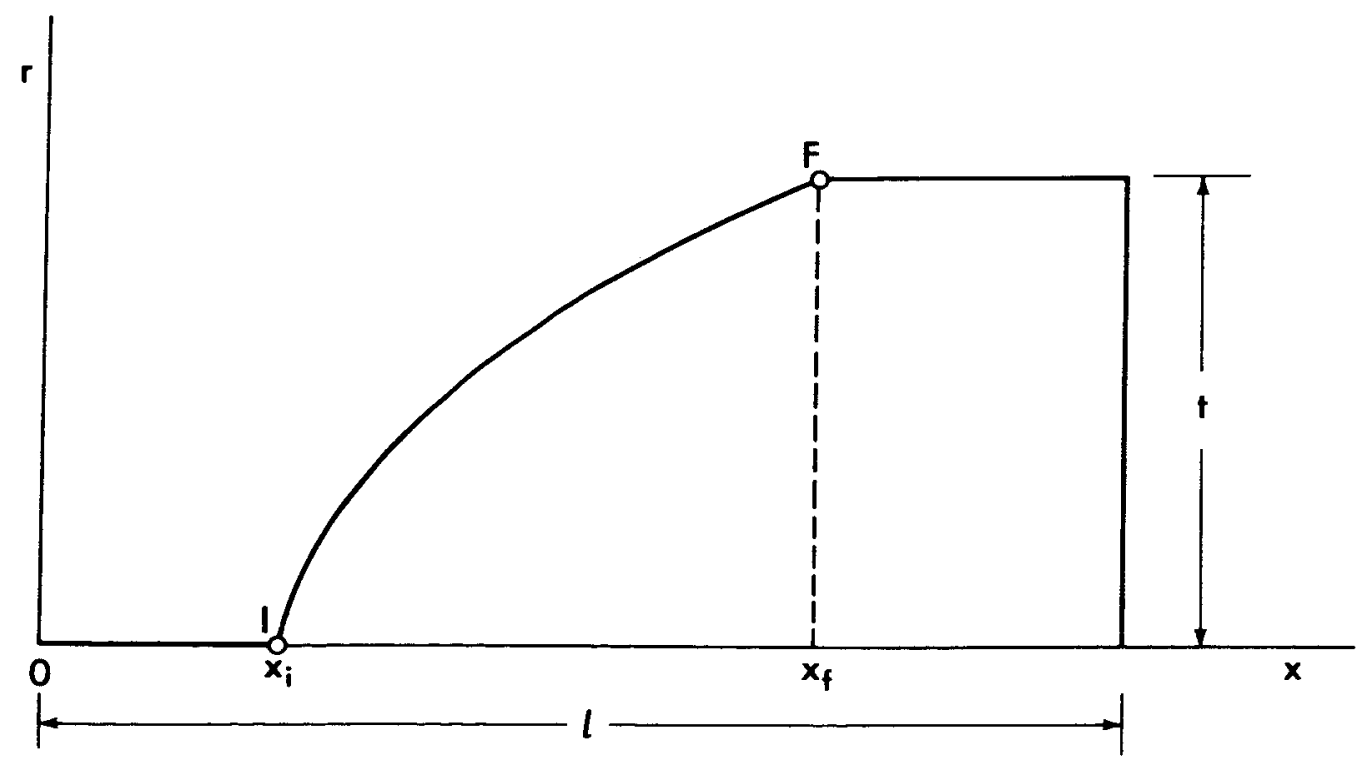

Fig. 2 Generalized meridian contour. 


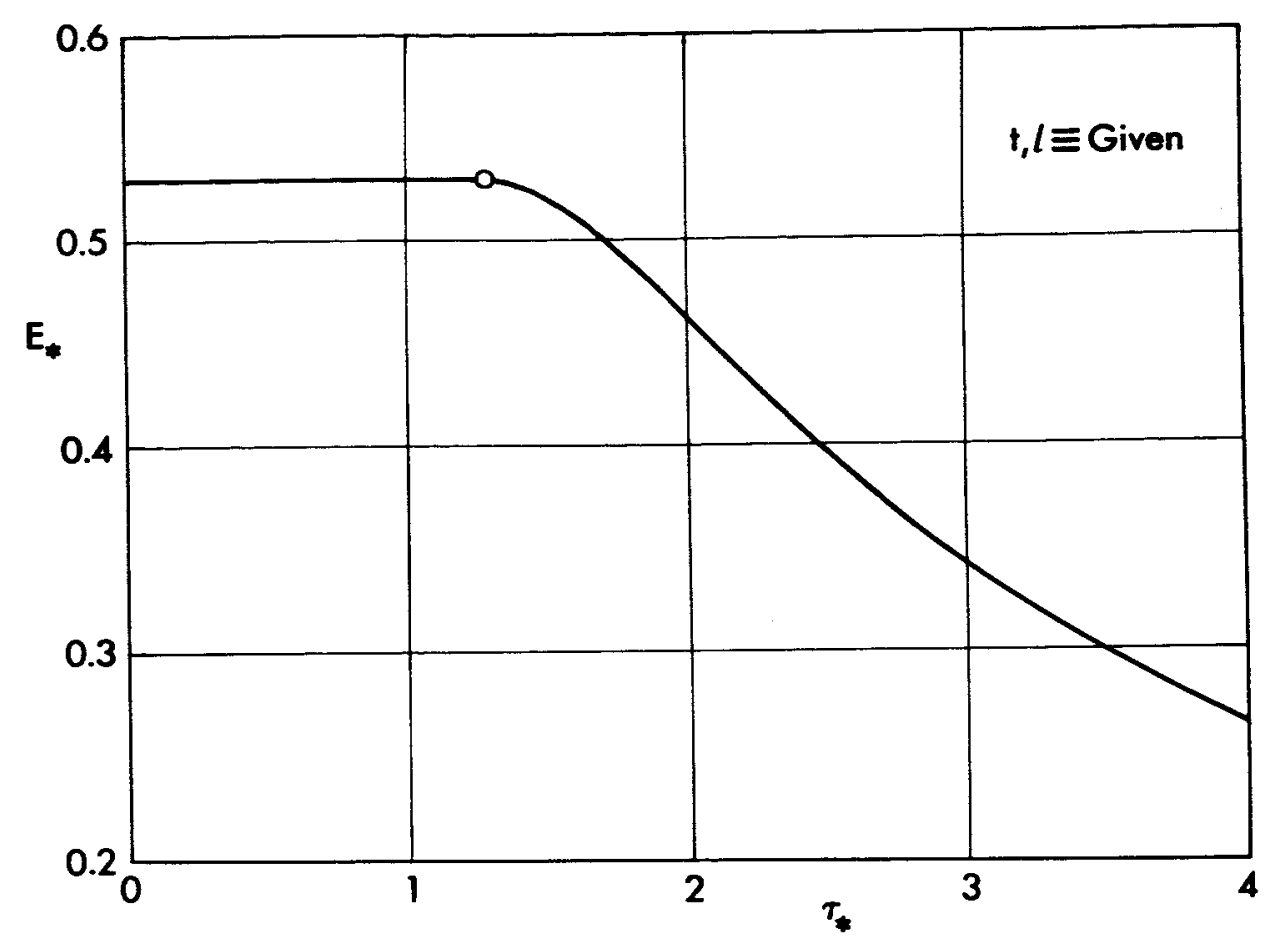

Fig. 3 Maximum lift-to-drag ratio.

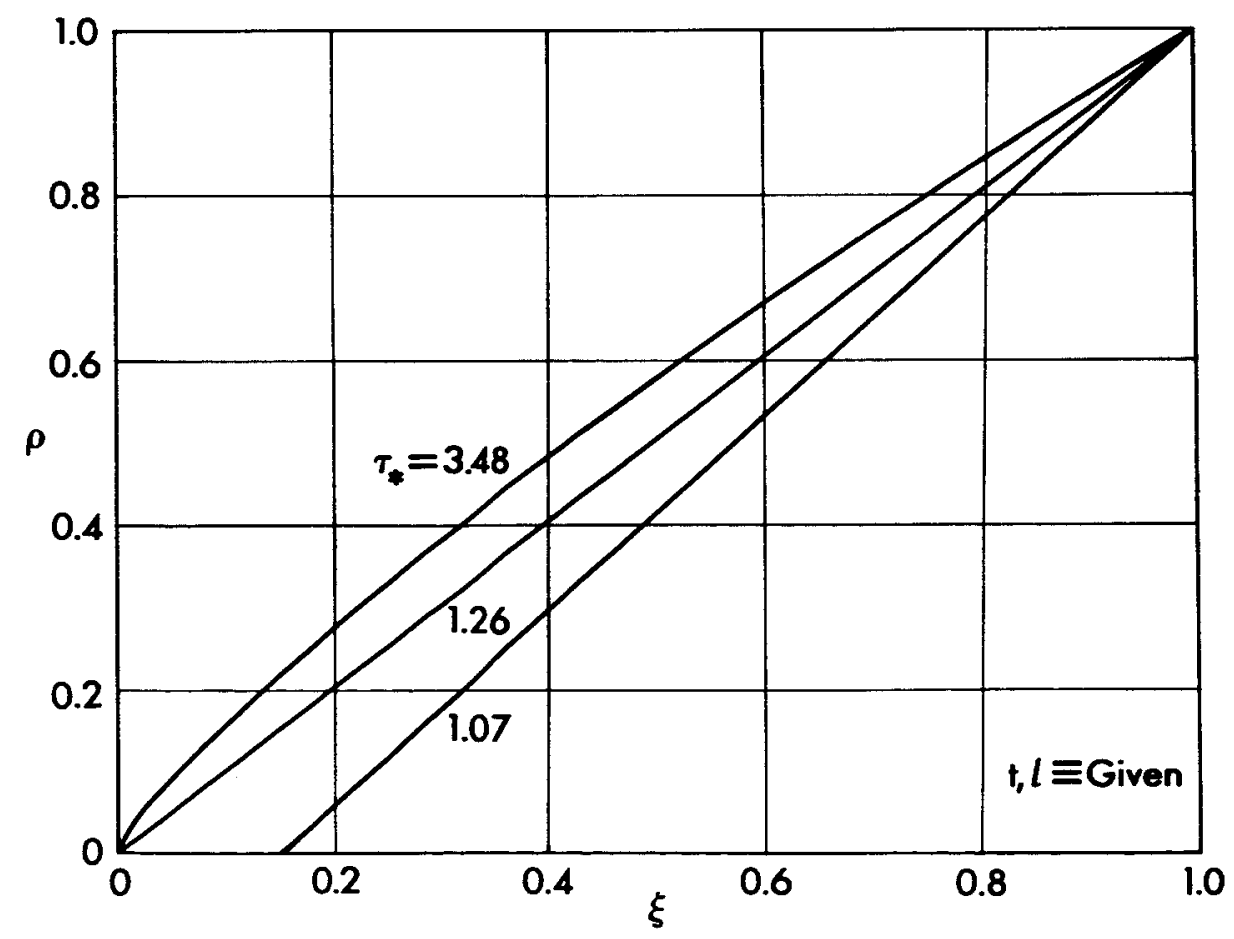

Fig. 4 Optimum shape. 


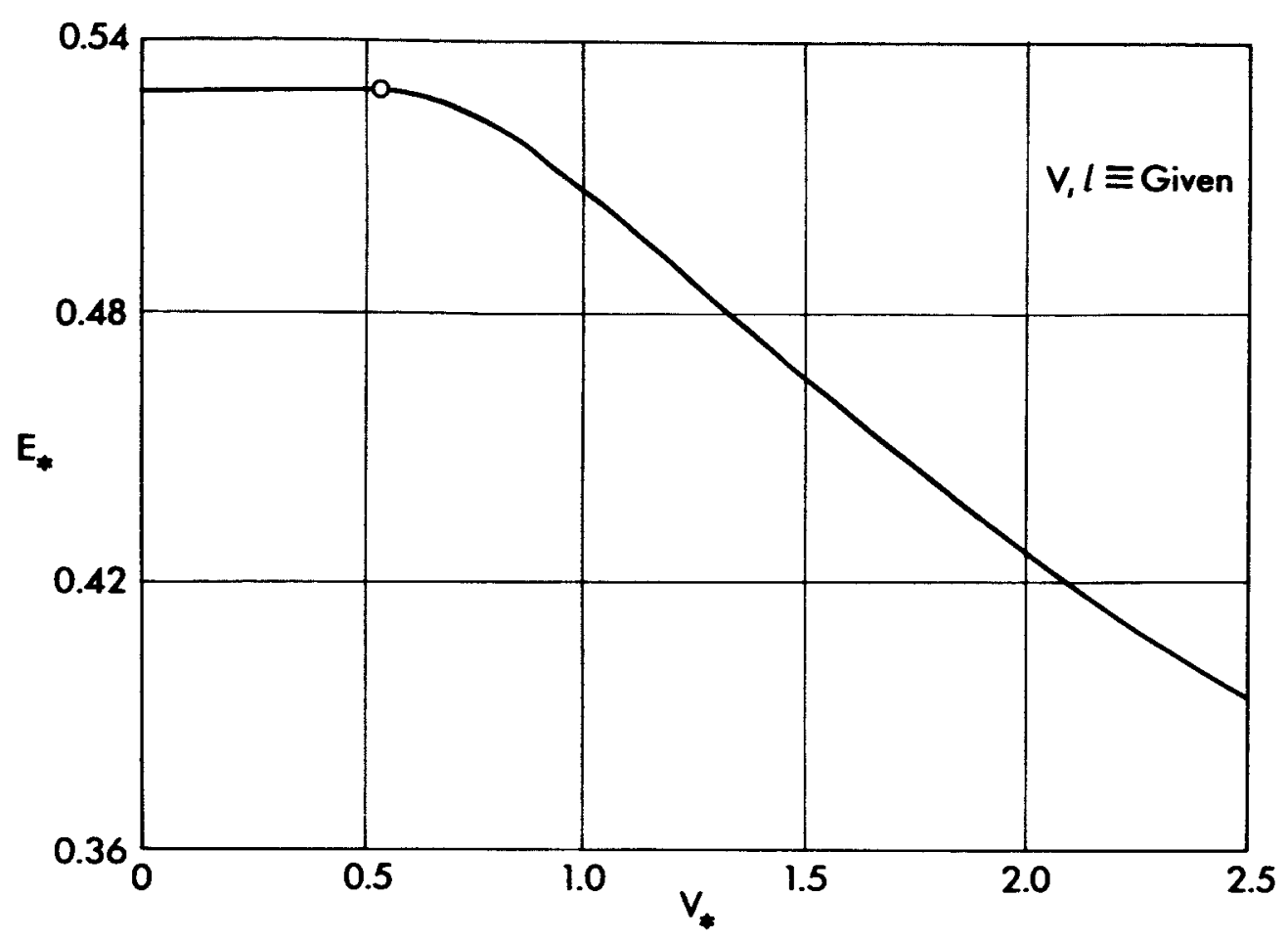

Fig. 5 Maximum lift-to-drag ratio.

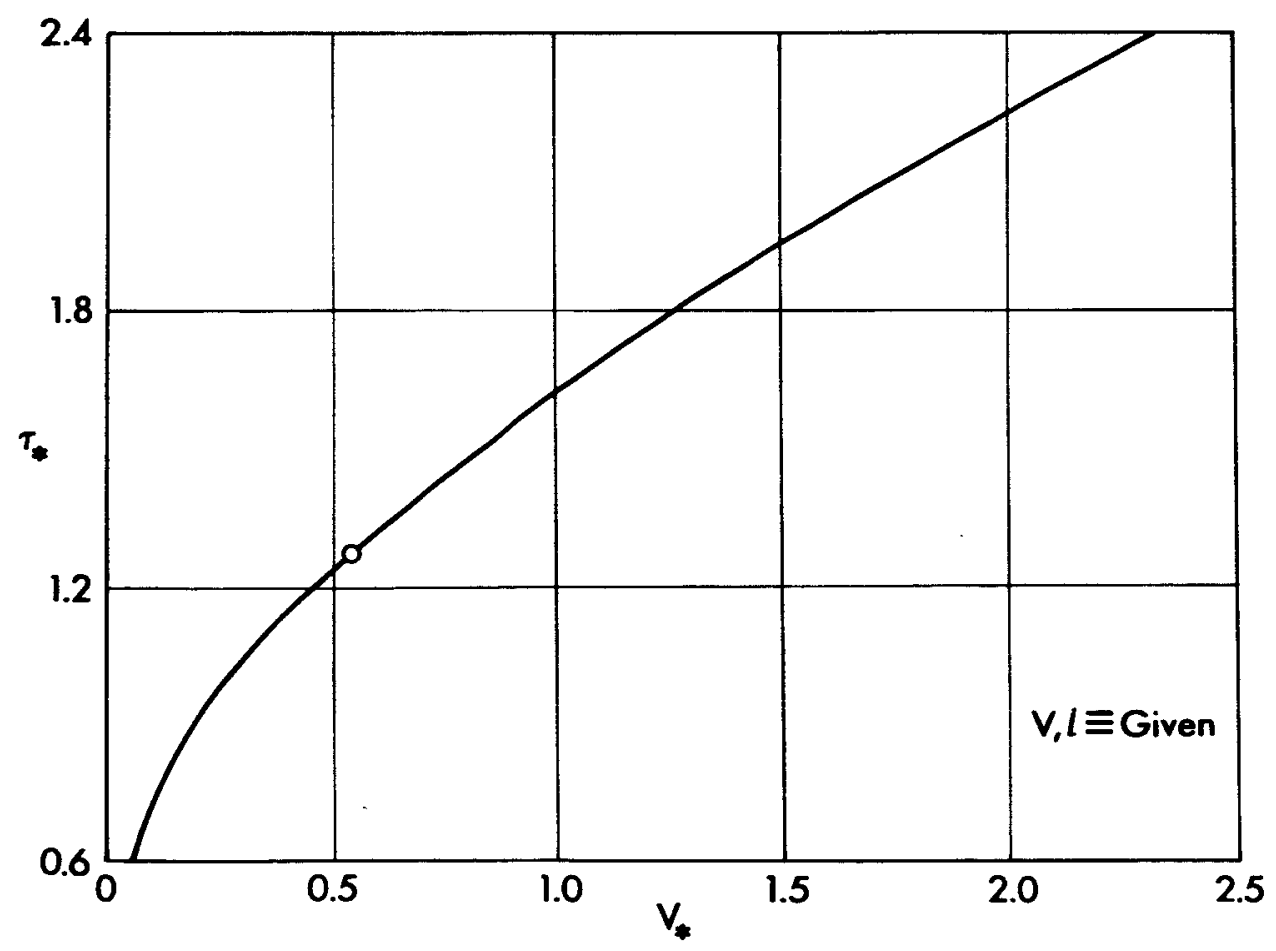

Fig. 6 Optimum thickness ratio. 


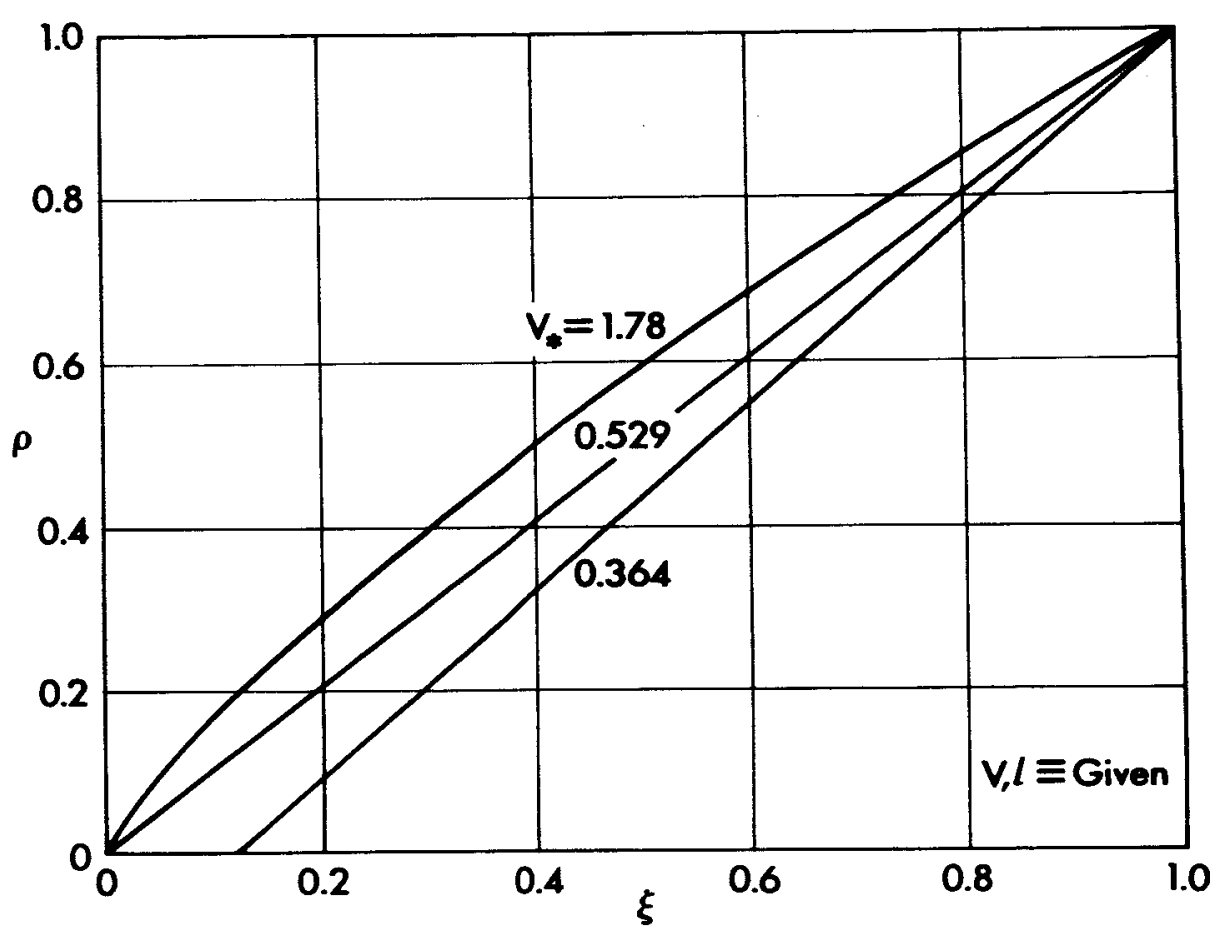

Fig. 7 Optimum shape. 


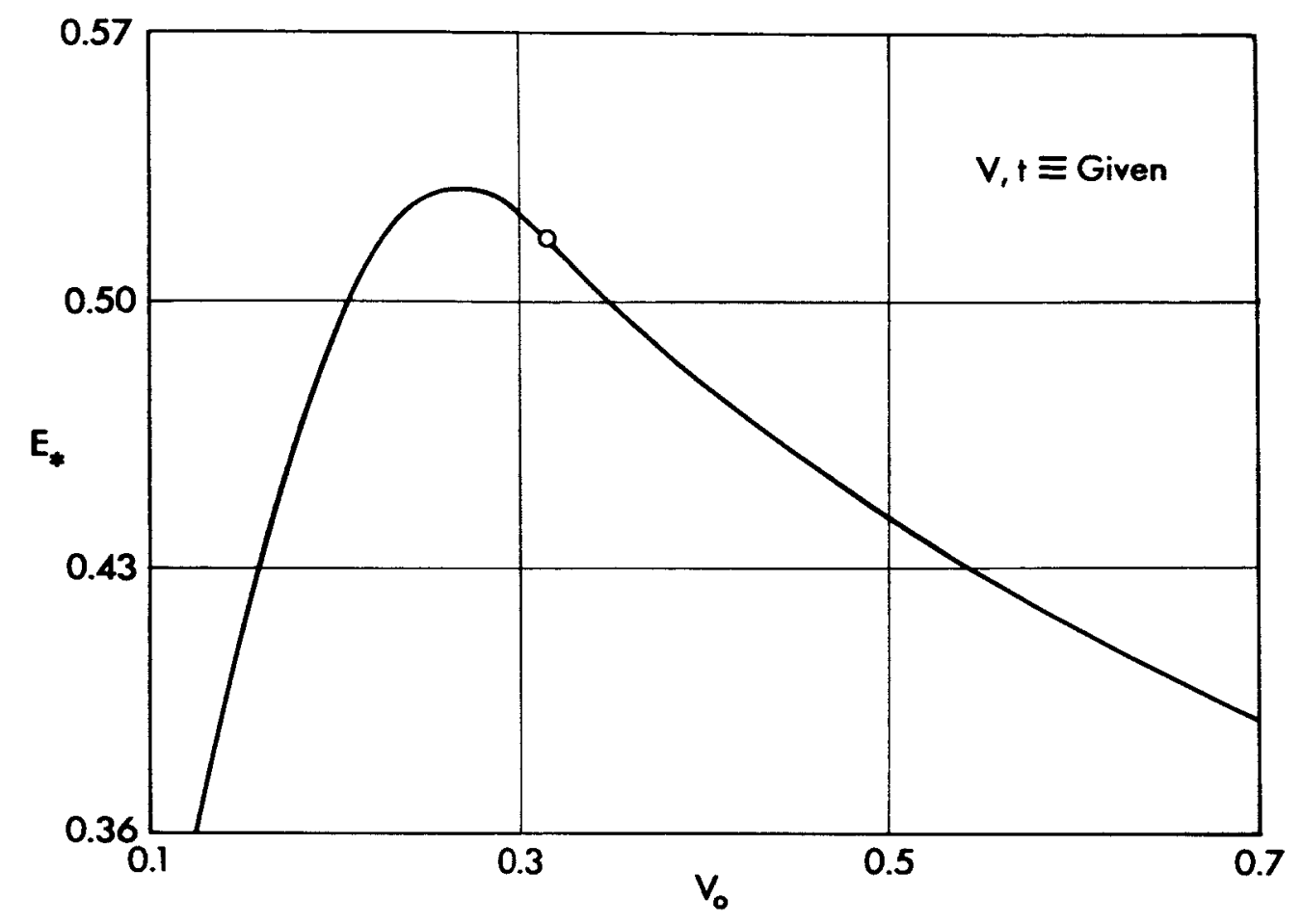

Fig. 8 Maximum lift-to-drag ratio.

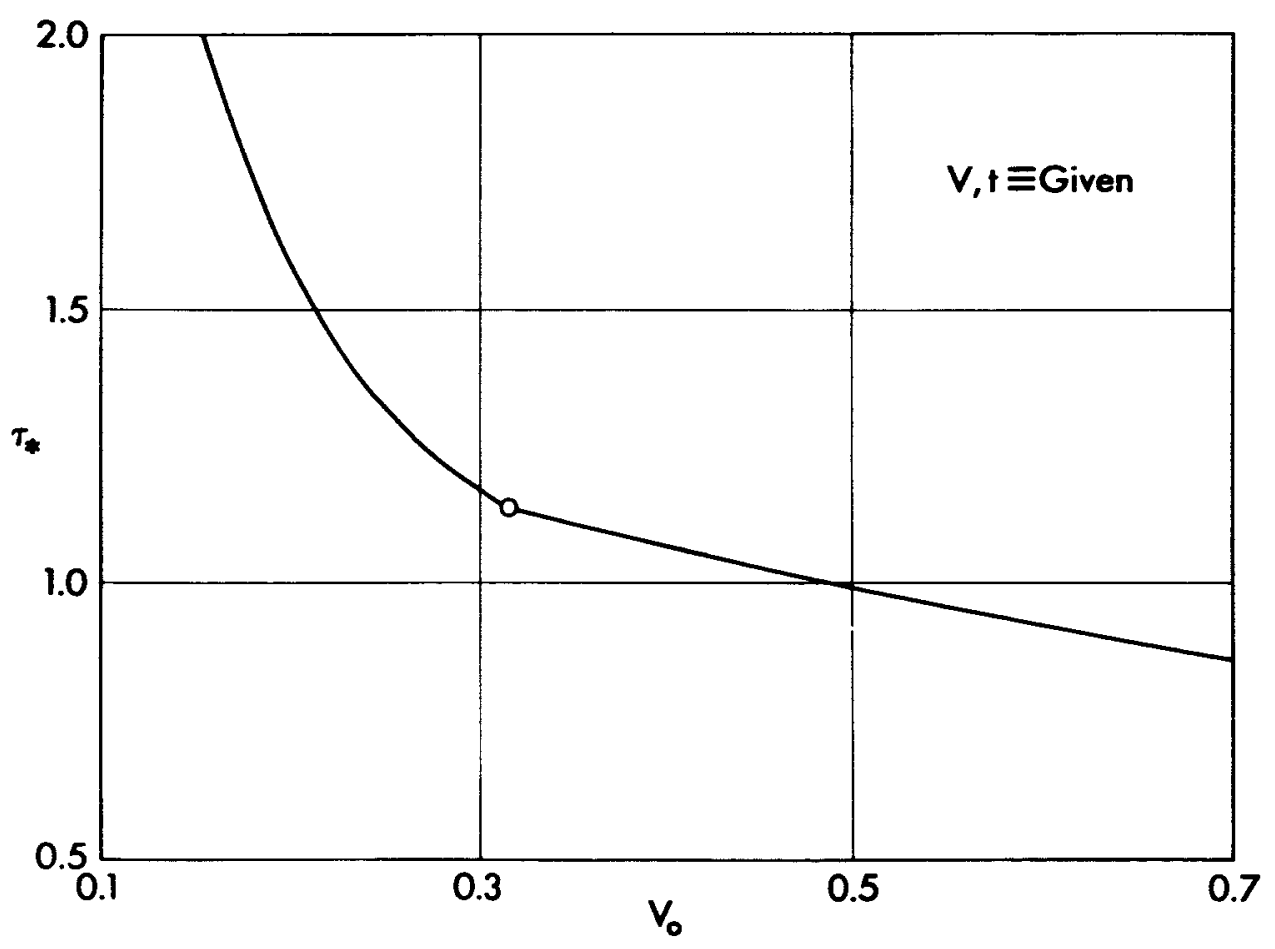

Fig. 9 Optimum thickness ratio. 


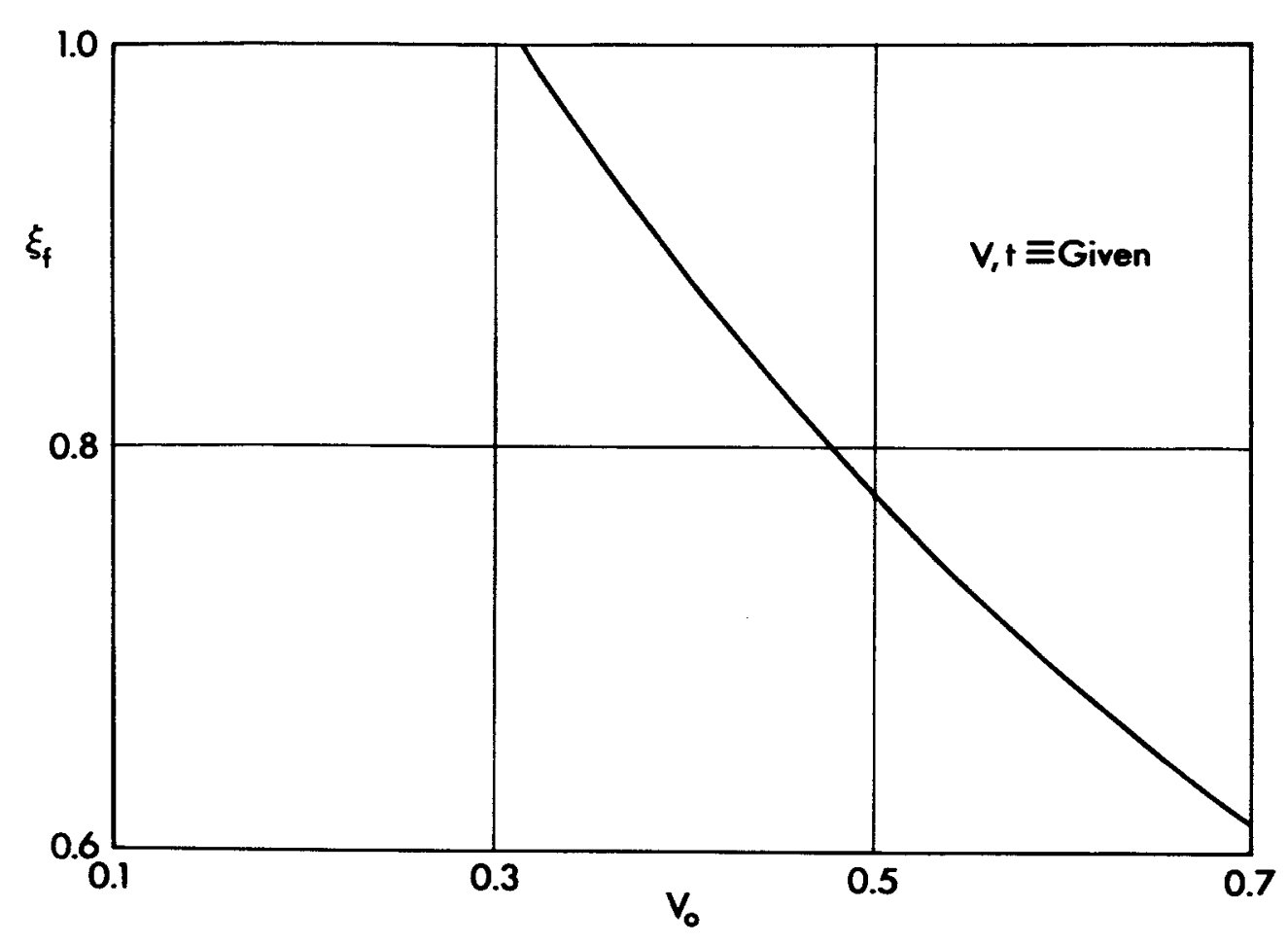

Fig. 10 Transition abscissa.

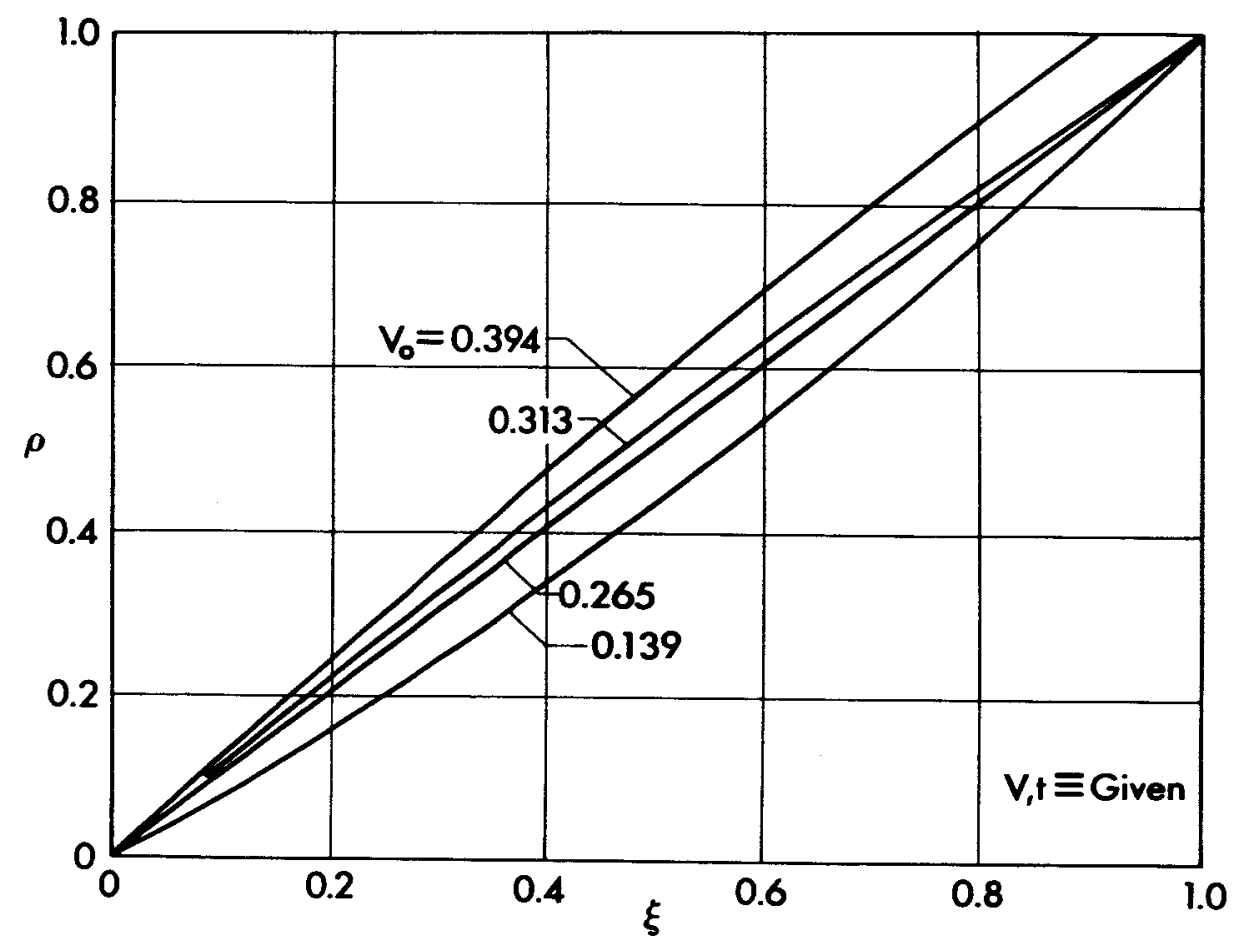

Fig. 11 Optimum shape. 